\title{
Impact of Multiresolution Segmentation Technique on Feature Extraction in Object Based Classification
}

\author{
Sherafgan Khattak \\ Department of Computer Systems Engineering, \\ University of Engineering \& Technology Peshawar, Pakistan. \\ sherafgan2006@gmail.com
}

\begin{abstract}
High-Resolution Imagery is futile until its spectral, spatial, and contextual properties are utilized. Object-based classification makes it possible with the help of powerful image segmentation (Multiresolution segmentation) algorithm. With the single segmentation level, it is difficult to extract all available features in a digital image. This research aimed to find the ideal parameter for extracting all features. And used those features for classifying the images (with the difference of a decade period) using two supervised classifiers, Fuzzy Nearest Neighbor and Support Vector Machine.Classificationresults showed that Scale parameter in multiresolution segmentation is directly proportional to the expandability of the image features. Moreover, it also showed that urban land has been increased in a decade period, while drastic reduction has occurred in green land. Correlating between two classifiers, SVM performed better with less execution time and higher overall accuracy of 0.98 and kappa statistics.
\end{abstract}

Of 0.97 .

Key words: Fuzzy Nearest Neighbor, Image Segmentation, Multiresolution Segmentation, Support Vector Machine.

\section{INTRODUCTION}

Currently, High-Resolution Imagery (HRI) is available to every researcher in remote sensing society. Having high spatial and spectral resolution [1], it can efficiently be used for generating precise land cover maps and extraction of different features. In past literature [2-3] traditional classification method reckons only on the pixel and its spectral values; which result is deplorable [4]. For maximum output, Object-Based Classification $(\mathrm{OBC})$ is considered a worthy tool [5]. However, feature selection and integration of training samples are still a critical task [6]. In OBC, image objects are formed using the segmentation process, which merges homogeneous pixels in terms of shape, color, texture, contextual information and their interrelationship. These image objects take part in further image analysis and classification [7]. Moreover, segmentation is an iterative process and it is an extremely difficult task to get, up to the mark segments [8,9]. Various segmentation algorithms i.e. Quadtree Segmentation, Chess-board segmentation, and Multiresolution segmentation are available in eCognition (Definiens cognition network technology). Among all, the Multiresolution segmentation is preferred for extraction of hidden features i.e. vegetation in barren hill and encroachment of green land. In $\mathrm{OBC}$, segmentation is directly proportional to better classification and feature extraction. On the same segmented HRI, two supervised classifiers have been correlated; one is Fuzzy Nearest neighbor (NN) and other is Support Vector Machine (SVM) in terms of their classification accuracy and kappa statistics. This research is aiming to use eCognition built-in image segmentation algorithm (Multiresolution segmentation) for extracting different features, classification of different land cover classes and the change occurred in a decade period of the same HRI.

\section{IMAGE SEGMENTATION}

Image segmentation is a process of segregating the entire digital image into segments and to classify these segments using classification methods [10]. Each segment is the combination of identical pixels in respect of texture, color and shape [11]. It is mostly used for identifying borders and curves in an image. Several approaches are brought under consideration based on its specific requirement, e.g. for finding the edges; edge detection techniques and for feature extraction; region-based segmentation is used [12].

\subsection{Multiresolution Segmentation}

Multiresolution segmentation (MRS) is a built-in image segmentation algorithm in eCognition. It starts working on considering a single pixel as an object along with its neighboring pixels and merges it based on the predefined criteria i.e. homogenous color, homogenous shape, texture and intensity [13] In OBC, these merged pixels are known as image objects. As image objects can be utilized for feature extraction and land cover classification, so MRS can be categorized as region-basedsegmentation. The size ofimage object is directly proportional to the user defined threshold, 
the Scale Parameter (SP); the higher SP leaves the algorithm to repeat several times to make larger image objects. Other parameters are shape(compactness, smoothness) andcolor. [14]. Various levels of segmentation process are needed till the findingof appropriate image objects; especially in feature extraction.

\section{STUDY AREA AND IMAGERY UTILIZED}

The focused area of this research is the urbanized area of Peshawar, Pakistan.SPOT-5 satellite imagery has been acquired from Pakistan Space Upper Atmosphere and Research Commission (SUPARCO) for the year 2005 and 2015 for more than 40 square kilometers. These imageshave four bands (Red, Green, Blue, and NIR) with $10 \mathrm{~m}$ spatial resolution.

\section{IMPLEMENTATION}

\subsection{Segmentation Measures}

Each SPOT-5 HRI (2005 and 2015) is processed in a separate eCognition project. And the same techniques, already discussed in section II are applied. According to the ground truth data, the total five featured classes including (urban land, green land, barren hill, bare land, and wetland) are identified. For these five classes, the images are segmented in three levels also shown in the table.1 but the segmentation result was similar for level 2 and level 3. This is because of the maximum capacity of the algorithm to merge the pixels. The segmentation results in the figures 1,2 and figures 3,4 for 2005 and 2015 respectively, clearly show that the objects in level 2 have large sizes as compared to the objects in level1.

Table 1: Multiresolution segmentation parameters for Level 1,2 and 3 respectively

\begin{tabular}{|l|l|l|l|l|}
\hline $\begin{array}{l}\text { Segmentation } \\
\text { Algorithm }\end{array}$ & Levels & $\begin{array}{l}\text { Scale } \\
\text { Parameter } \\
\text { (SP) }\end{array}$ & Color & Shape \\
\cline { 4 - 5 } & Level1 & 16 & 0.8 & $0.4 / 0.2$ \\
\cline { 2 - 5 } $\begin{array}{l}\text { Multiresolution } \\
\text { Algorithm }\end{array}$ & Level2 & 40 & 0.7 & $0.7 / 0.3$ \\
\cline { 2 - 5 } & Level3 & 80 & 0.6 & $0.8 / 0.4$ \\
\hline
\end{tabular}

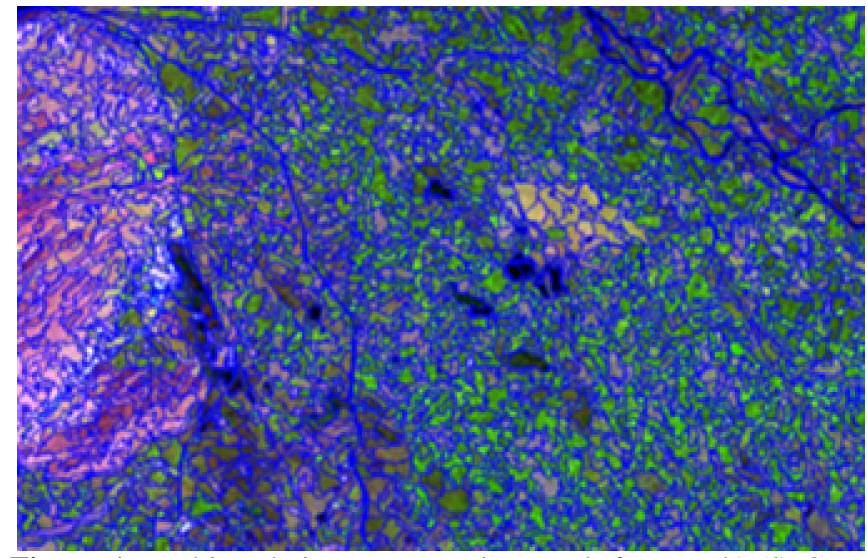

Figure 1: Multiresolution segmentation result for Level1 (SPOT-5 2005)

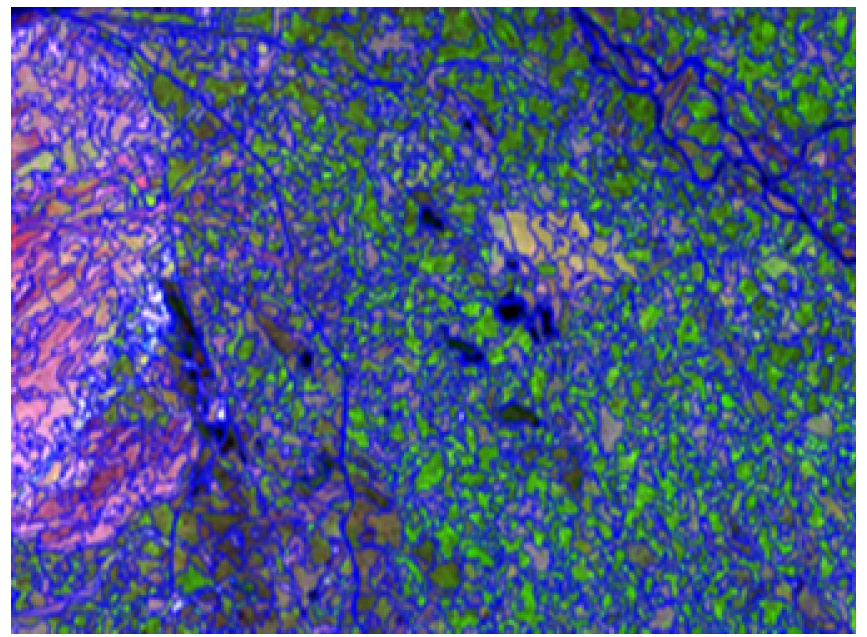

Figure 2: Multiresolution segmentation result for Level2 (SPOT-5 2005)

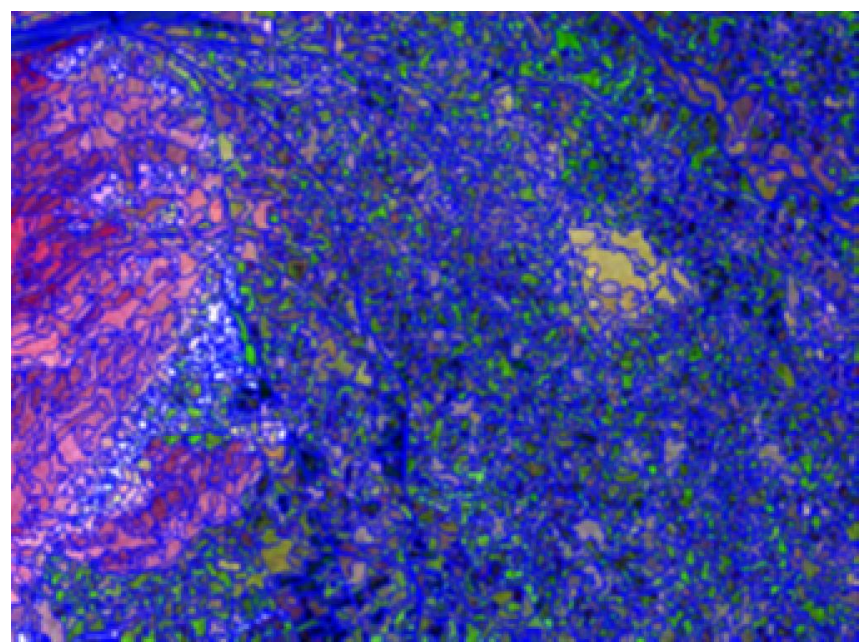

Figure 3: Multiresolution segmentation result for Level1 (SPOT-5 2015) 


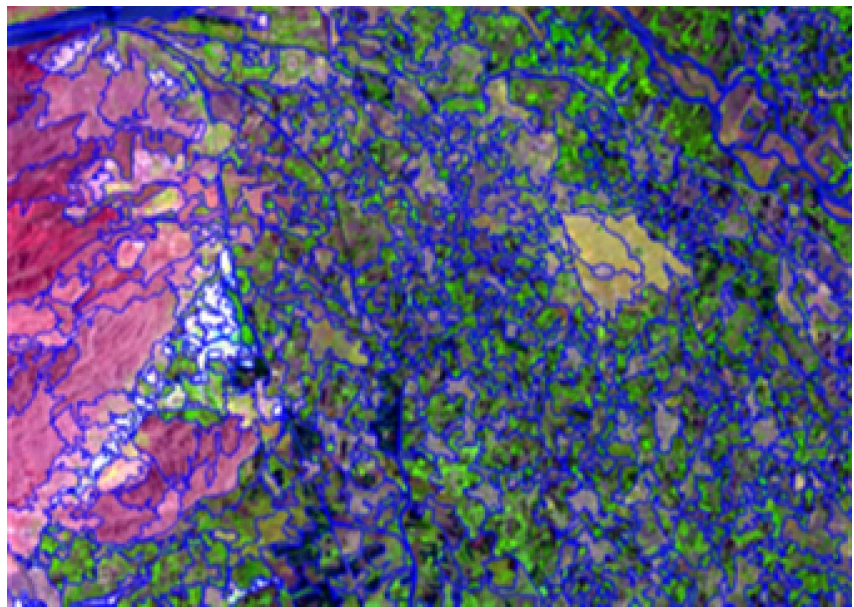

Figure 4: Multiresolution segmentation result for Level2 (SPOT-5 2015)

\subsection{Supervised Classifiers}

In implementation, the second phase is to classify the segmented images of the first phase into the five land cover classes i.e. urban land, green land, barren hill, bare land and wetland. For classification, two supervised classifiers fuzzy NN and SVM are utilized. NNworks on mean of two neighbors in the entire image [15]. While SVM works on hyperplane and kernel [16].Classification results in figures 5, 6 and figures 7, 8 for 2005 and 2015 of NN and SVM are shown respectively. Urban land is displayed in red, bare land in yellow, barren hill in white, green land in green and wetland in blue color.

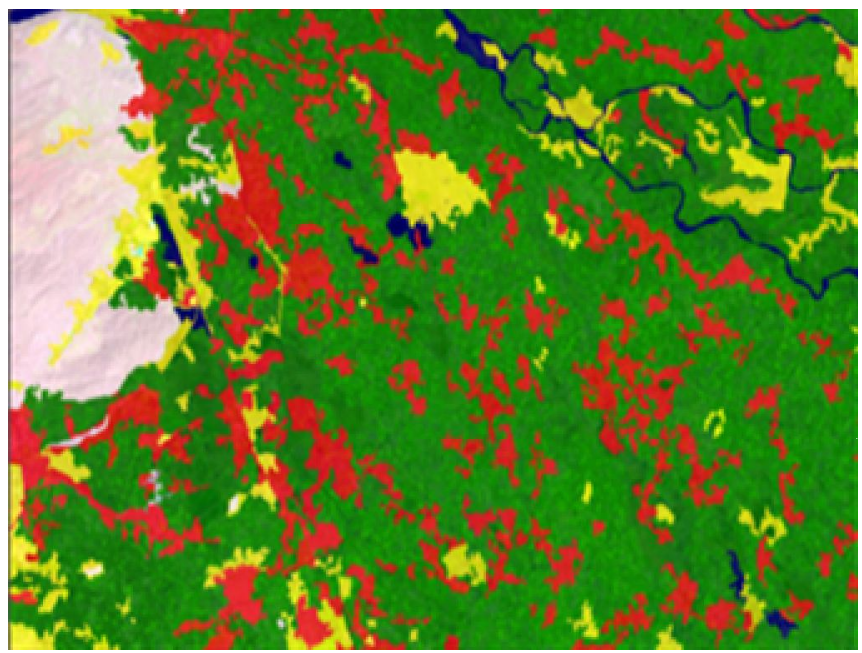

Figure 5: Classification of (SPOT-5 2005) image by Nearest Neighbor

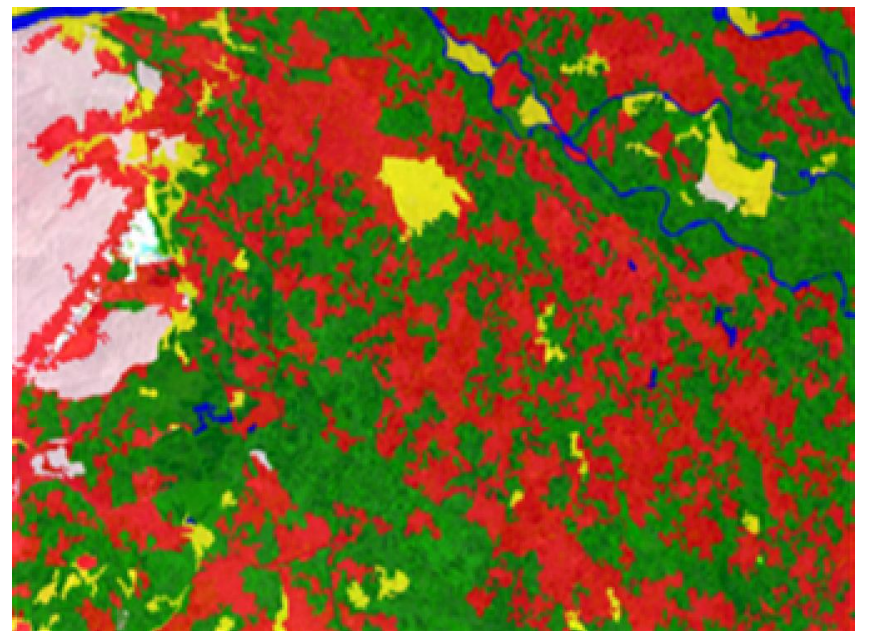

Figure 6: Classification of (SPOT-5 2015) image by Nearest Neighbor

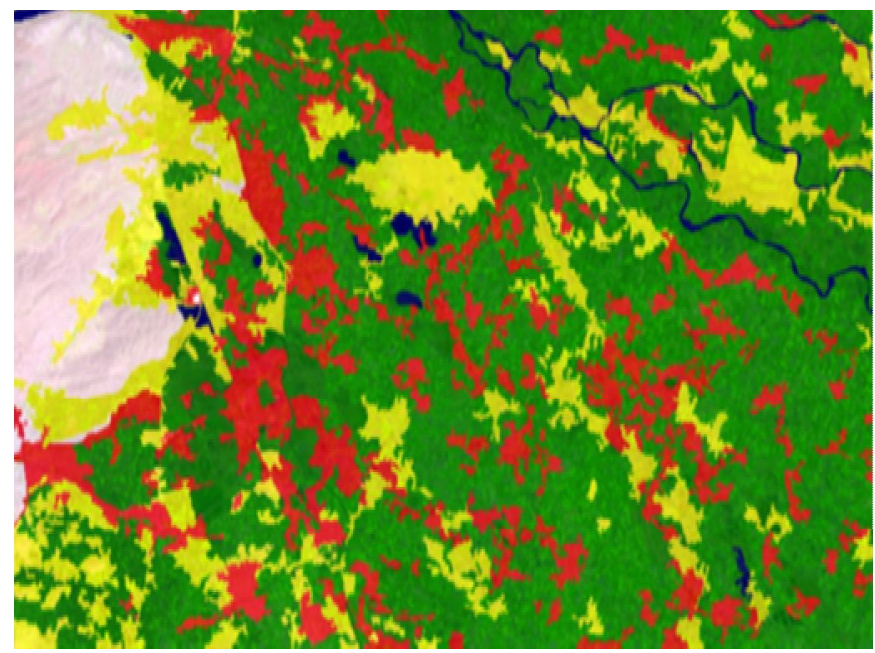

Figure 7: Classification of (SPOT-5 2005) image by Support Vector Machine

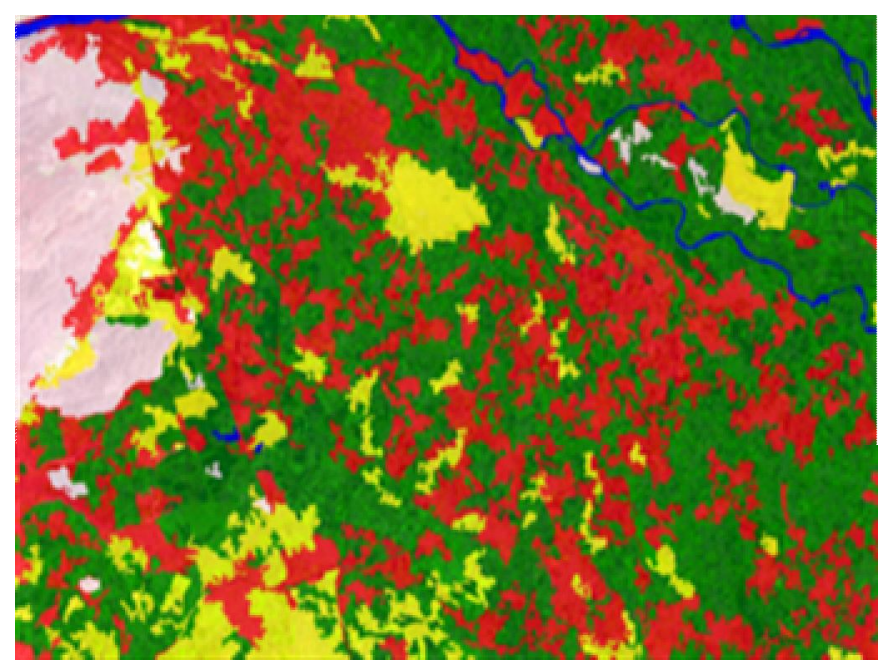

Figure 8: Classification of (SPOT-5 2015) image by Support Vector Machine 


\section{RESULTS AND ACCURACY CALCULATION}

Confusion matrix also known as error matrix and Kappa statisticsalso known as Cohen's kappa are used to calculate the accuracy. The confusion matrices are calculated in every classifier for every image also shown in table 2 . The detailed misclassification is also generated on every execution of the classifier, but those misclassifications are removed by incorporating user ground truth experience into sample selection process. Here overall accuracy and kappa statisticsare used to correlate both classifiers. In both classifiers, Wetland feature has extracted normally for any segmentation level. The green land area is expanded, coversa large area in 2005 image, so high SP value will result in large objects. Thus, for extracting expanded objects, high SP values are required which is also shown in figure 5 and 7 . In 2015, urban land is expanded, thus in level 2 of 2015, also shown in figure 6 and 8, the urban land is classified in a change profound manner covering large area. Comparing with the ground truth data, the huge change occurred in a decade period in Peshawar, also shown in table 3 and figures 9 and 10. Correlating both classifiers, the SVM values are promising.

Table: 2: Error Matrix (Overall Accuracy and Kappa Statistics of NN and SVM)

\begin{tabular}{|l|l|l|l|l|}
\hline Years & \multicolumn{2}{|l|}{$\mathbf{2 0 0 5}$} & \multicolumn{2}{l|}{2015} \\
\\
\hline Classes & NN & SVM & NN & SVM \\
\hline Overall Accuracy & 0.962 & 0.978 & 0.96 & 0.983 \\
\hline Kappa Statistic & 0.961 & 0.972 & 0.96 & 0.98 \\
\hline
\end{tabular}

Table: 3: Difference in objects with respect to time from 2005 to 2015 (NN and SVM)

\begin{tabular}{|l|l|l|l|l|l|l|l|l|l|l|l|}
\hline \multirow{2}{*}{ Years } & \multicolumn{2}{|c|}{ B.H } & \multicolumn{2}{|c|}{ W.L } & \multicolumn{2}{|c|}{ U.L } & \multicolumn{2}{|c|}{ B.L } & \multicolumn{2}{|c|}{ G.L } \\
\cline { 2 - 11 } & $\mathbf{N}$ & $\mathbf{S}$ & $\mathbf{N}$ & $\mathbf{S}$ & $\mathbf{N}$ & S & N & S & N & S \\
\hline 2005 & 66 & 68 & 20 & 21 & 198 & 182 & 144 & 170 & 425 & 413 \\
\hline 2015 & 53 & 55 & 18 & 18 & 329 & 289 & 112 & 139 & 287 & 298 \\
\hline $\begin{array}{l}\text { \% } \\
\text { Differ } \\
\text { ence }\end{array}$ & 19 & -23 & \begin{tabular}{l}
11 \\
\hline
\end{tabular} & 16 & +39 & +35 & -28 & -22 & -48 & -38 \\
\hline
\end{tabular}

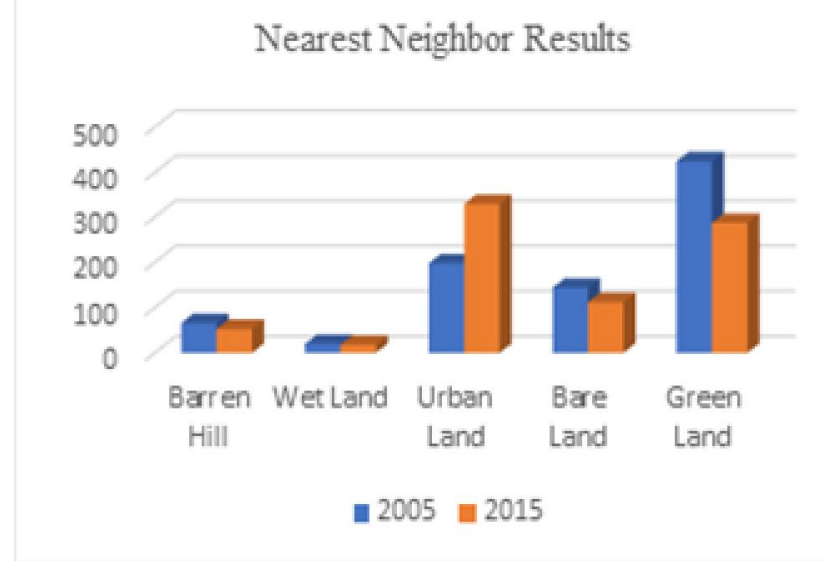

Figure 9: Graphical representation of difference in objects with respect to time from 2005 to 2015 (NN)

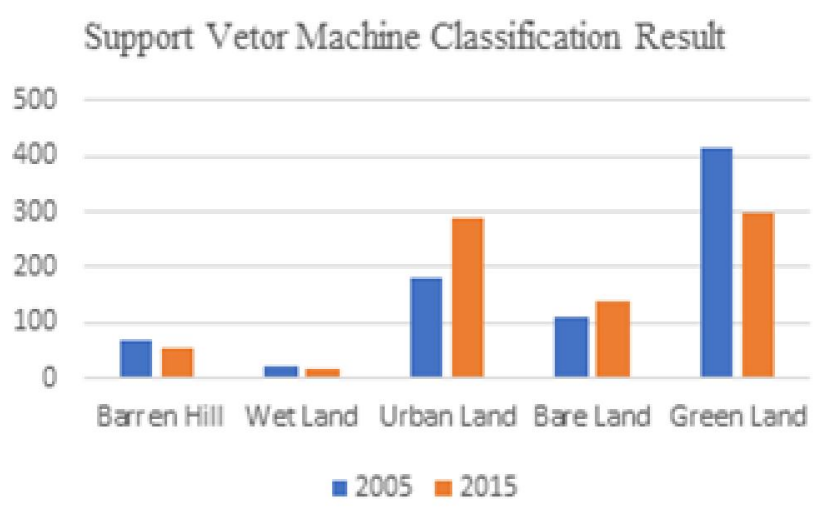

Figure.10: Graphical representation of difference in objects with respect to time from 2005 to 2015 (SVM)

\section{CONCLUSION}

In this research, the object-based classification on the Highresolution imagery has been utilized. The Image segmentation and particularly Multiresolution segmentation has implemented on SPOT-5 Satellite imagery. It is pertinent to note that the image segmentation was worth using for HRI due to the utilization of all its properties in a good manner. Moreover, the image features have a direct connection with the scale parameter of the MRS. And multiple features can be extracted by varying the scale parameter value along with the shape and compactness. Between the two classifiers, SVM performed better, in its execution time and results. The urban land has drastically increased while the green land has decreased.

\section{REFERENCES}

1. Jiang, N., et al. "Semi-automatic building extraction from high resolution imagery based on segmentation." 2008 International Workshop on Earth Observation and Remote Sensing Applications. IEEE, 2008. https://doi.org/10.1109/EORSA.2008.4620311 
Sherafgan Kattak, International Journal of Advances in Computer Science and Technology, 8(3), March 2019, 16 - 20

2. Ahmed et al., "Comparing Pixel-Based Classifiers for Detecting Tobacco Crops in North-West Pakistan," in Recent Advances in Space Technologies (RAST), 2015 7th International Conference on, 2015, pp. 211-216: IEEE. https://doi.org/10.1109/RAST.2015.7208343

3. Expansion and Land Use Changes In Akure, Nigeria, Using Remote Sensing And Geographic Information System (GIS) Techniques," vol. 4, no. 9, pp. 533-541, 2011.

4. Xiaoxia, Sun, Zhang Jixian, and Liu Zhengjun. An objectoriented classification method on high resolution satellite data. CHINESE ACADEMY OF SURVEYING AND MAPPING BEIJING (CHINA), 2004.

5. T. Blaschke, "Object Based Image Analysis for Remote Sensing," ISPRS Journal of Photogrammetry and Remote Sensing, vol. 65, no. 1, pp. 2-16, 2010. https://doi.org/10.1016/j.isprsjprs.2009.06.004

6. S. Laliberte, J. Koppa, E. L. Fredrickson, and A. Rango, "Comparison of nearest neighbor and rule-based decision tree classification in an object-oriented environment," in Proceedings of IEEE international Geoscience and Remote Sensing Symposium, Denver, Colorado, USA, 2006, pp. 3923-3926.

https://doi.org/10.1109/IGARSS.2006.1006

7. Wong, T. H., et al. "Feature extraction based on objectoriented analysis." Proceedings of ATC 2003 Conference. Vol. 2021. 2003.

8. Dey, Vivek, Yun Zhang, and Ming Zhong. "A review on image segmentation techniques with remote sensing perspective." (2010): 31-42.

9. Wen Zhou, Dongping Ming, Lu Xu, Hanqing Bao, and Min Wang, "Stratified Object-Oriented Image Classification Based on Remote Sensing Image Scene Division," Journal of Spectroscopy, vol. 2018, Article ID 3918954, 11 pages, 2018.

10. Ajay Kumar Thakur, Madhusudan, Image Segmentation: A Comparative study, International Journal of Advances in Computer Science and Technology, Volume 5, No.6, June 2016, 64-71

11. Kaur, Harmanpreet. "Review of remote sensing image segmentation techniques." International Journal of Advanced Research in Computer Engineering \& Technology (IJARCET) 4.4 (2015): 1667-1674.

12. U. C. Benz, P. Hofmann, G. Willhauck, I. Lingenfelder, and M. Heynen, "Multi-Resolution, Object-Oriented Fuzzy Analysis of Remote Sensing Data For GIS-Ready Information," ISPRS Journal of Photogrammetry and Remote Sensing, vol. 58, no. 3-4, pp. 239-258, 2004. https://doi.org/10.1016/j.isprsjprs.2003.10.002

13. Trimble, "eCognition-ReferenceBook," 2012.
14. Darwish, K. Leukert, and W. Reinhardt, "Image Segmentation for The Purpose of Object-Based Classification " in Geoscience and Remote Sensing Symposium, 2003. IGARSS'03. Proceedings. 2003 IEEE International, 2003, vol. 3, pp. 2039-2041: Ieee.

15. Iman, Paryudi,Alternative Design Exploration using $\mathrm{K}$ Nearest Neighbor Technique andSemantic Web Technology in an Energy Simulation Tool, International Journal of Advances in Computer Science and TechnologyVolume 2, No.10, October 2013.

16. Tzotsos and D. Argialas, "Support Vector Machine Classification for Object-Based Image Analysis," in ObjectBased Image Analysis: Springer, 2008, pp. 663-677. 